\title{
Sending a message to the other side
}

\author{
A range of mechanisms have evolved for communicating information across cell membranes, but \\ designing synthetic analogues is far from trivial. A collection of articles in this issue discuss different \\ methods of passing chemical information across lipid bilayers using artificial systems.
}

The cell membrane provides a physical barrier that separates the internal contents of a cell from the surrounding environment. This separation enables the generation of higher (or lower) concentrations of chemicals within the cell compared to the extracellular conditions. Furthermore, it enables the formation of electrochemical gradients across the cell membrane that can be used to drive biological processes ${ }^{1}$. It also means that reactions can be conducted under different conditions to those found outside of the cell and this can lead to altered reactivity or provide protection from extracellular compounds that would otherwise interfere.

However, cells also need to be able to sense and respond to changes in their environment and a range of mechanisms have evolved that can detect chemical cues, communicate the detection into the cell and provide an output that can trigger a downstream response. These processes are impressive and complex, but researchers wanting to go beyond what nature has provided - such as adding new functionalities to synthetic cells or developing new bioreactors that can sense and respond to the external environment will need to develop artificial systems. And the intricate nature of signal transduction and transmembrane transport machinery means that repurposing biological systems is far from simple.

One route for chemical signals to cross a cell membrane is for ions to pass through a membrane-spanning pore ${ }^{2}$. In an Article in this issue on page 411 , a team led by Hagan Bayley and Derek N. Woolfson describe an artificial transmembrane pore self-assembled from $\alpha$-helical peptides. Constructing a channel within the hydrophobic environment of a lipid bilayer is challenging because the hydrophobic interactions between helices that are required to assemble a viable pore can be disrupted. To overcome this problem the team drew inspiration from nature and based their design on the C-terminal domain of an Escherichia coli polysaccharide transporter. After identifying a suitable peptide sequence they demonstrate that the assembled pore is functional and capable of conducting ions across a membrane.
G-protein-coupled receptors found in cell membranes detect molecules outside the cell and - by undergoing a conformational change - transmit this information and activate a signal cascade inside the cell ${ }^{3,4}$. In an Article on page 420 a team led by Jonathan Clayden and Simon J. Webb report their efforts to replicate this behaviour in a synthetic system, whereby ligand binding at one end of a receptor results in a conformational change that leads to a detectable output at the other. Previously, Clayden and co-workers have shown that a photoresponsive foldamer can undergo conformational switching between left and right helices when bound in a phospholipid bilayer $^{5}$. In the present study they demonstrate that the helix switching of a related foldamer - which has a metal-centred binding site at one end - is triggered by binding a ligand in the aqueous phase on one side of a bilayer. This conformational change propagates along the length of the foldamer and results in a change in the fluorescence of pyrene groups at the other end of the molecule.

Adopting a different approach, a team led by Christopher A. Hunter and Nicholas H. Williams set out to develop an artificial signal transduction pathway based on translocation of a synthetic molecule across a cell membrane (page 426). The transducer molecule is embedded within the membrane but is too short to span it, and so sending a chemical message requires it to move within the membrane. To trigger this translocation, the transducer has two headgroups at either end that can protrude into the aqueous phase. An input signal switches the polarity of one of the head-groups, resulting in movement of the transducer across the membrane. Another feature that is commonly found in biological signal transduction pathways is an amplification step that magnifies the initial chemical response ${ }^{6,7}$. Hunter and co-workers designed their system so that the second internal head-group is a pro-catalyst. Once the second head-group enters the aqueous phase on the opposite side of the bilayer it binds a $\mathrm{Zn}^{2+}$ ion, thereby turning on catalytic activity - resulting in a proportionally greater chemical output.

Although the artificial systems reported by Clayden/Webb and Hunter/Williams operate through different mechanisms, they both aim to detect a chemical signal and subsequently report its presence remotely without directly transporting the chemical entity responsible for triggering the initial signal. An accompanying News \& Views article by Scott L. Cockroft on page 406 contrasts the differences between the two approaches and briefly discusses some of the wider implications of these results.

Finally, in an Article on page 431 a team led by Edward S. Boyden showcase how transporting chemical information across the lipid bilayer in synthetic minimal cells can enable genetic circuits to be controlled by chemical cues. Mutually incompatible reaction networks cannot operate within the same nanoreactor and so they need to be physically separated; however, this separation also prevents interactions between the different networks - such as one network modulating the other. Using a mixed population of liposomes that encapsulate different reaction networks Boyden and co-workers report the communication of chemical information between different synthetic minimal cells. The team also go on to demonstrate how controlled fusion of liposomes can be used to combine reaction networks that are initially separated. This approach enables the products of incompatible reactions to be brought together and encapsulated within a single nanoreactor.

There are still many challenges that need to be overcome when developing new synthetic systems capable of transmitting a chemical signal across a cell membrane or lipid bilayer. Nevertheless, these studies show some of the ways in which researchers can repurpose natural systems and even go beyond biological principles - and such systems may provide the basis for a range of chemical applications in the future.

\footnotetext{
References

1. Stryer, L. Biochemistry 3rd edn (W. H. Freeman, 1988).

2. Rosenberg, S. S. \& Spitzer, N. C. Cold Spring Harb. Perspect. Biol. 3, a004259 (2011)

. Manglik, A. \& Kobilka, B. Curr. Opin. Cell Biol. 27, 136-143 (2014).

4. Venkatakrishnan, A. J. et al. Nature 494, 185-194 (2013).

De Poli, M. et al. Science 352, 575-580 (2016).

6. Pierce, K. L., Premont, R. T. \& Lefkowitz R. J. Nat. Rev. Mol. Cell Biol. 3, 639-650 (2002).

7. Willoughby D. \& Cooper D. M. F. Physiol. Rev. 87, 965-1010 (2007).
} 